Universidad de Lima

Facultad de Ciencias Empresariales y Económicas

Carrera de Marketing

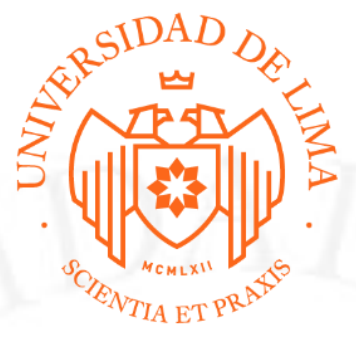

\title{
ESTUDIO SOBRE LA SITUACIÓN DE LA EMPRESA DICOMTECH
}

Trabajo de suficiencia profesional para optar el Título Profesional de Licenciado en Marketing

\section{IVO CARLOS RAMOS}

Código 20131672

\author{
Asesor \\ Alfredo José Galdo Jiménez
}

Lima - Perú

noviembre de 2018 


\section{ESTUDIO SOBRE LA SITUACIÓN DE LA EMPRESA DICOMTECH}




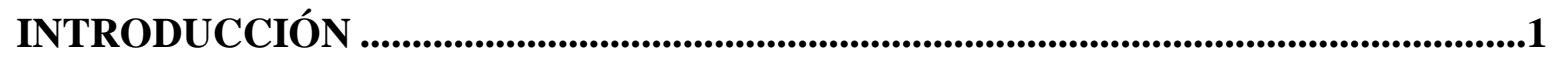

CAPÍTULO I: CONTEXTO DE LA EMPRESA............................................................3

1.1 Descripción y antecedentes de la industria en la que se desempeña la empresa ............3

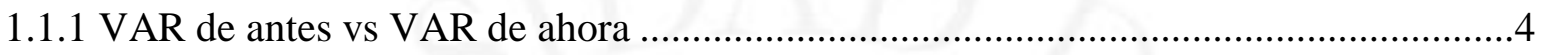

1.1.2 Tendencias que moldean el mercado VAR .................................................................6

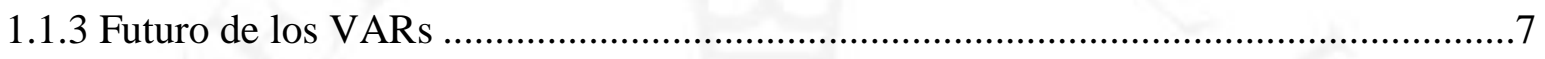

1.1.4 Industria TI en el Perú .....................................................................................

1.2 Descripción de la empresa en cuanto a los accionistas, estructura organizacional, unidades de negocios, marcas y mezcla de productos ...........................................................

1.2.1 Accionistas

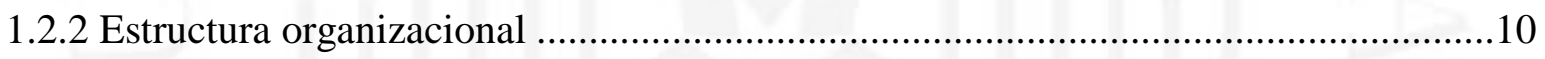

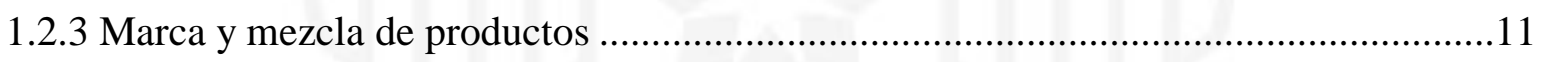

1.3 Descripción del mercado y sus segmentos ............................................................15

1.4 Cuantificación de la industria en volumen de unidades y/o valor de la venta.............16

1.5 Identificación, descripción y participación de mercado de competidores directos .....17

CAPÍTULO II: DIAGNÓSTICO DEL MARKETING ESTRATÉGICO DE LA

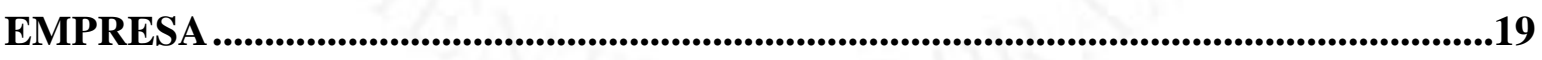

2.1 Descripción y análisis de la estrategia genérica............................................................19

2.2 Descripción y análisis de la(s) ventaja(s) competitiva(s) ............................................20

2.3 Descripción y análisis de la(s) estrategia(s) de marketing corporativo que aplica ......20

2.4 Descripción y análisis de la(s) estrategia(s) competitiva(s) de marketing que aplica .21

2.5 Descripción del(los) grupo(s) objetivo que atiende .....................................................22 
2.6 Descripción del posicionamiento de la empresa o marca(s) ..................................23

2.7 Descripción de la creación del Brand Equity de su(s) marca(s) .............................25

CAPÍTULO III: DESCRIPCIÓN DEL CASO PROBLEMA ............................................29

3.1 Descripción de la situación ........................................................................29

CAPÍTULO IV: ANÁLISIS Y DISCUSIÓN DE RESULTADOS .....................................32

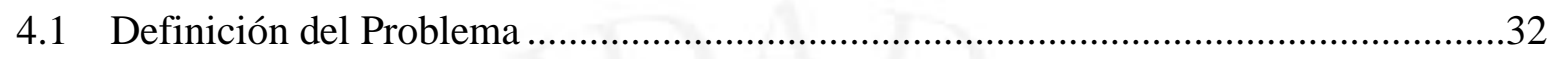

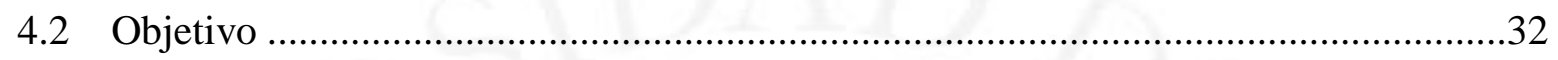

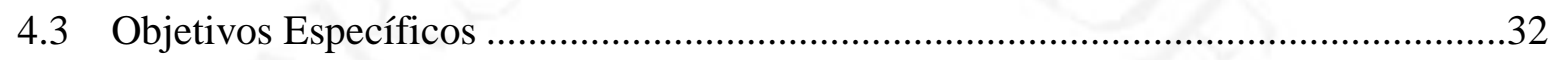

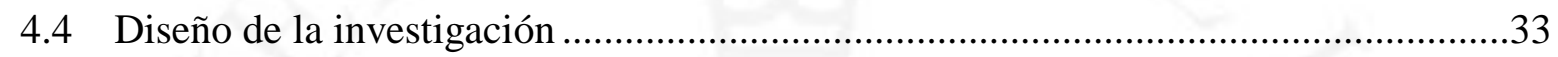

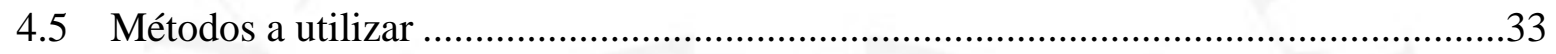

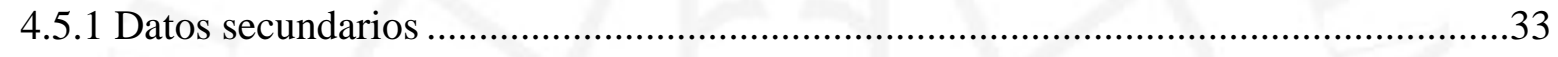

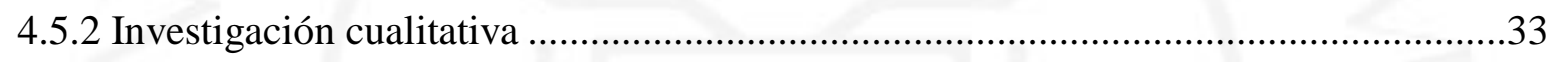

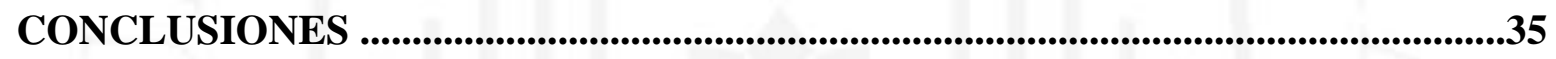

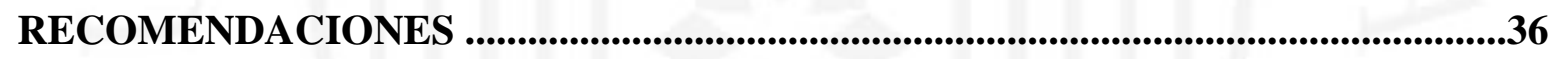

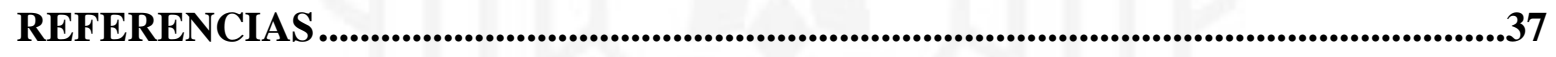

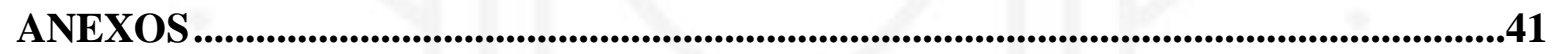




\section{ÍNDICE DE TABLAS}

Tabla 1.1.1 Sectores de la industria TI ................................................................. 8

Tabla 1.2.1 Cartera de productos ManageEngine ............................................................. 12

Tabla 1.5.1 Ranking de empresas por facturación...................................................... 18

Tabla 2.6.1 Posibles Propuestas de valor ............................................................. 24 


\section{INDICE DE FIGURAS}

Figura 1.1.1 Dos tipos de canales de distribución .................................................. 4

Figura 1.2.1 Estructura organizacional de Dicomtech............................................... 10

Figura 1.2.2 Ejemplo de lanzamiento de producto ................................................... 13

Figura 1.2.3 Ejemplo de versiones producto OpManager .......................................... 14

Figura 1.3.1 Perú: Empresas, según segmento empresarial, 2015-16 …........................ 15

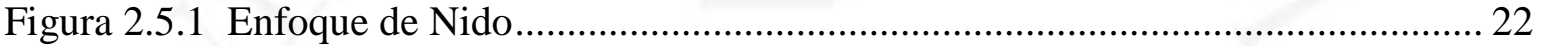

Figura 2.7.1 Logo Dicomtech .............................................................................. 26

Figura 2.7.2 Campaña de email marketing ............................................................... 27

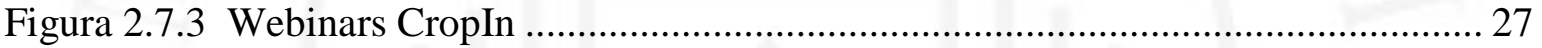

Figura 2.7.4 Seminario de seguridad de TI y Active Directory....................................... 28

Figura 3.1.1 Precios de paquetes OpManager .......................................................... 30

Figura 3.1.2 Detalles de la estructura de licencia de OpManager Plus ............................ 30 


\section{INDICE DE ANEXOS}

Anexo 1: Guía de indagación a expertos .............................................................. 42

Anexo 2: Guión y pautas de Mystery Shopper. ............................................................... 44

Anexo 3: Cotización SolarWinds ................................................................................ 46 


\section{INTRODUCCIÓN}

Dicomtech empieza en el 2010 como una empresa de servicios de outsourcing de personal

de TI, posteriormente, se agregan una gama de herramientas de tecnologías que acompañarían al servicio y lo convertirían en una mejor propuesta de valor para el cliente. Es de esta manera como Dicomtech en el 2011 se asocia con el desarrollador de softwares de administración integral de TI, ManageEngine, para poder soportar los servicios de consultoría y adicionalmente, ofrecer sus herramientas tecnológicas a potenciales clientes del mercado peruano.

Destaca principalmente por el staff de técnicos compuesto por profesionales altamente capacitados y muy competitivos con amplia experiencia de trabajo en empresas mineras, petroleras, consumo masivo, etc., así como en entornos internacionales, multiculturales y diversos. El detalle más importante es que tanto el gerente general como el gerente de sistemas están capacitados en la India, país de procedencia del desarrollador de software ManageEngine.

La razón por la cual ambos gerentes fueron capacitados en la India se debe al conjunto de requerimientos que se debió cumplir para ser autorizado como "Partner". ManageEngine pidió a Dicomtech alcanzar a clientes nacionales con una oferta de valor agregado, certificaciones técnicas a través de programas de entrenamiento y colaboración con la realización de eventos. A cambio de cumplir estos objetivos, Ellos también darían apoyo de corte financiero, publicitario, entre otros. 
Uno de los problemas identificados fue el tema de precio. La "P" del marketing mix antes mencionada es la que más intriga al gerente general pues en sus propias palabras: "Ofrecemos Productos de alta calidad, pero a un bajo precio pues son desarrollados en la India. No cabe duda de la calidad mostrada por ManageEngine como transnacional y el talento de nuestro Staff Técnico, es por eso que estoy seguro de que se puede aumentar hasta 30 por ciento el precio e incluso ser muy bajo respecto a la competencia que manejan precios de industria relativamente elevados".

La solución planteada es un plan de investigación de mercado ajustado a los requerimientos y limitaciones de la compañía como pequeña empresa pero que ya cuenta con una prestigiosa cartera de clientes. 


\section{CAPÍTULO I: CONTEXTO DE LA EMPRESA}

\subsection{Descripción y antecedentes de la industria en la que se desempeña la empresa}

Dicomtech se desempeña en la industria de TI (Tecnologías de información) que en rasgos generales seria la aplicación de ordenadores y equipos de telecomunicación para almacenar, recuperar, transmitir y manipular datos. Específicamente, Dicomtech vendría a ser un VAR (Value-AddedReseller), es decir, un revendedor de valor agregado.

Una empresa VAR se entiende como una compañía que revende software, hardware y productos de networking y provee un valor agregado que va por encima de la orden de compra (AllBusiness Editors, s.f.). En el caso de Dicomtech, un ejemplo podría ser el sistema de administración de Active Directory mejorado con otro producto de la cartera como OPManager, el cual se encarga de la administración de servidores que contienen al Active Directory. Dentro del paquete que puede ofrecer un VAR aparte de la venta del hardware y software están: el planeamiento estratégico, diseño de sistema, implementación, entrenamiento, trazado de activos, soporte técnico, desarrollo de bases datos, consultoría y búsqueda. (AllBusiness Editors, s.f.)

Son estos añadidos lo que permite a los VARs diferenciarse de los proveedores de productos, como es el caso de los retailers. Además, le da la oportunidad al cliente de integrar una variedad de servicios que necesite de una misma fuente, en vez de solicitarlo a varios proveedores. Se debe acotar que un VAR no es un (AllBusiness Editors, s.f.):

- Retailer: Los distribuidores minoristas generalmente añaden poco valor al producto.

- Proveedor de soluciones e integrador de sistemas: Son organizaciones grandes que se asemejan a los VARs pero que enfrentan proyectos más complejos y largos.

- Proveedores de servicios de aplicación: Administran y entregan soluciones de software vía internet, por lo que solo se necesita dumbterminals (monitor con poca o nula capacidad de procesamiento) o computadoras de bajo precio. 
Dicomtech como se mencionó anteriormente, cuenta con la ventaja de trabajar directamente con el fabricante de IT, no siendo el único, mientras que otros VARs solo pueden conseguir el producto mediante un distribuidor del fabricante como lo muestra el siguiente cuadro:

Figura 1.1.1

Dos tipos de canales de distribución

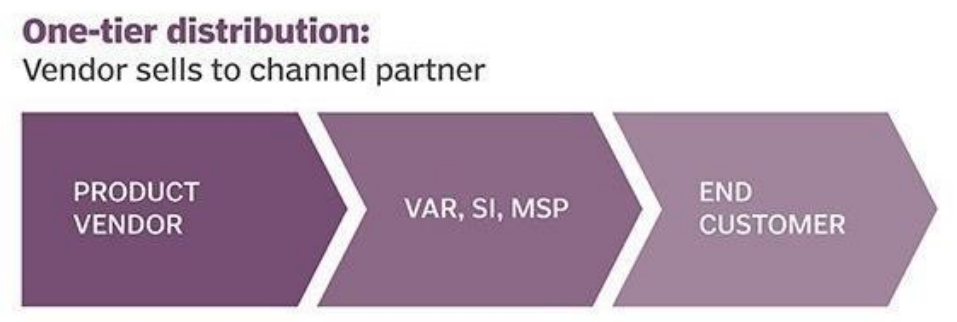

\section{Two-tier distribution:} Vendor sells to distributor, which sells to channel partner

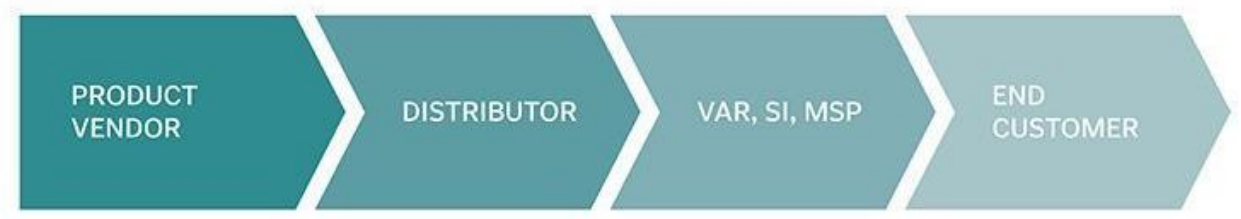

Fuente: TechTarget (2018)

\subsubsection{VAR de antes vs VAR de ahora}

Los inicios de los VARs estuvieron marcados por la introducción de tecnologías no tan masificada como lo es en la actualidad referente a las TI específicamente. Hoy por hoy, existe un peligro en la industria debido a la erosión de su propuesta de valor causada por la introducción de alternativas como servicios más económicos gracias al cloud y hardware pre configurado, el acceso a los precios y paquetes disponibles en internet y la integración vertical de los fabricantes de software. (MacLean, 2012) 
La fortaleza de los VARs en los 90 recaía en la falta de información de los consumidores respecto a la integración del software y el hardware y a la falta de opciones de proveedores (MacLean, 2012). El rol de los VARs también pasa por la cobertura de áreas que el equipo de ventas de los fabricantes no puede alcanzar, como logística, mantenimiento, asesoría, integraciones, entre otros y que les permitía fungir como una extensión del equipo de TI del desarrollador. (Gillis, 2014)

El panorama actual es diferente pero no nuevo. La tendencia al uso de cloud se venía acercando desde el 2008 como se mostraba en la revista Connected donde se hablaba sobre el cambio que iban a experimentar los VARs hacia los manage services (servicios gestionados) (Roy et al., 2008). Esto último cambio radicalmente las reglas del juego, donde el consumidor es protagonista y decisor en tiempo real de los cambios que se generan en su empresa.

Los managed services es la práctica de una empresa a

Entregar parcial o total la administración de su infraestructura de red, aplicaciones y seguridad a los expertos en redes. Estos expertos pueden incluir proveedores de servicios, integradores de sistemas o revendedores de valor agregado. Las empresas tienen la opción de comprar un servicio o conjunto de servicios administrados específicos o un paquete todo incluido que incluye la mayoría de las funciones de TI. (ACG Research, s.f., párr. 1)

Los manages services son entonces la tercerización de la gestión TI de una empresa que permite mantenerse a la vanguardia y estar al día en cuestiones de software. Estos servicios responden a la necesidad del cliente de flexibilidad para adoptar y deshacerse de nueva tecnología a su placer, un precio mensual con el que puedan trabajar en sus modelos operativos, y la libertad de toda responsabilidad que conlleva poseer una infraestructura tecnológica. (Blackmon, 2017) 
Esto último no necesariamente responde al comportamiento de las medianas y grandes empresas, sin embargo Dicomtech está al tanto de las tendencias que en los próximos años llegarían con más fuerza, en tanto los servicios cloud se consoliden y exista una confianza más marcada. Imaginar a grandes entidades como Interbank o Saga deshacerse de su estructura de IT parece aun algo increíble, pero tal como demostró Alibaba o Amazon, la realidad está más

cerca.

\subsubsection{Tendencias que moldean el mercado VAR}

Las tendencias que se observan en el transcurso de los últimos años no han permutado de forma considerable como para agregar más a su conjunto. El estudio sobre los jugadores claves en el mercado de revendedores de software muestra lo que la empresa Dicomtech ha experimentado con el pasar de los años (Jones y Roelevel, 2011).

- Los desarrolladores de software refinan sus programas de canales para crear relaciones más íntimas con los VAR: Dado que el objetivo son las ventas. los fabricantes ofrecen incentivos financieros y certificaciones para ayudar al revendedor con la tarea de ventas, y más aún, con la promoción de sus productos por encima de los competidores. Esto es especialmente cierto en Dicomtech, donde se capacito técnicamente a los actores principales de la empresa para que vendieran más fácilmente su cartera de productos.

- Los desarrolladores regionales se alían para expandir su alcance global: Las alianzas permiten a los VARs proporcionar un servicio más íntimo en la lengua local, que si aperturaran sucursales en todos los países vecinos desde un HUB regional.

- Los grandes VARs subcontratan especialistas pequeños. Ya que varias compañías prefieren hacer negocios con uno o pocos revendedores por simplicidad. Las VARs de mayor tamaño subcontratan o compran productos de otros revendedores especializados para entregar una solución completa mientras se sigue negociando con una sola empresa. Subcontratar en más fácil que crear alianzas, las cuales toman tiempo y son difíciles de terminar; el inconveniente para los subcontratistas es si hay muchas 


\subsubsection{Futuro de los VARs}

Como se comentó anteriormente, el presente más obvio de los VARs será el de adoptar los servicios cloud como parte de su oferta pero también la infraestructura convergente (CI) (Gillis, 2014), la cual:

Empaqueta los componentes básicos (computación / servidores, almacenamiento de datos, equipos de red y la virtualización y otro software para la administración de infraestructura) en unidades integradas y optimizadas. CI llega al sitio ya configurado, probado y certificado, por lo que las soluciones de CI se instalan y mantienen fácilmente. CI va de la mano con la virtualización: los recursos de computación se agrupan, comparten aplicaciones y otras cargas de trabajo, y se optimizan con la ayuda de un supervisor y otro software de control (Custom Solutions Group,s.f., párr. 1)

Es en palabras simples la agrupación de tecnologías de información en un único y optimizado paquete computacional para la administración de los recursos de TI. Se debe recordar que la aplicación de estas tecnologías requerirá mayores habilidades que están más asociadas a los integradores de sistemas que a los VARs menciona Sainsbury, SVP de Dimensions Data Americas (Blackmon, 2017).

Dado que el mercado exige a los VARs que se incorporen nuevos servicios a su portafolio, esto también implica el desarrollo de nuevas competencias para prosperar en los años que siguen. Los softwares, en particular, impulsaran la necesidad de desarrollar una serie de habilidades para mantener al negocio a la par de la evolución tecnológica. (Blackmon, 2017). "El más grande desafío de los revendedores viene por su sustitución en la cadena de distribución producto de las nuevas alternativas que no precisan de ellos" (MacLean, 2012,p.23). La única manera de mantenerse será la de adoptar las nuevas tecnologías mientras en el Perú aun éstas no están consolidadas.

\subsubsection{Industria TI en el Perú}

Debido a la naturaleza de la industria TI en el Perú, existen varios sectores en los que puede ser desglosada como se especifica en el siguiente cuadro: 
Tabla 1.1.1

Sectores de la industria TI

\begin{tabular}{|c|c|}
\hline Sector & \multicolumn{1}{|c|}{ Descripción } \\
\hline \multirow{2}{*}{ Bienes } & $\begin{array}{l}\text { Producción de bienes de consumo de T.I. tales como equipos } \\
\text { informáticos y de telecomunicaciones digitales, bienes de capital (por } \\
\text { ejemplo, maquinaria automatizada para la fabricación de Computadoras } \\
\text { Personales) y bienes intermedios tales como placas base, discos duros, } \\
\text { unidades de DVD, entre otros periféricos, utilizados en la fabricación de } \\
\text { computadoras. }\end{array}$ \\
\hline Software & $\begin{array}{l}\text { Diseño, producción, mantenimiento y comercialización de } \\
\text { software empaquetado y/o personalizado. }\end{array}$ \\
\hline Infraestructura & $\begin{array}{l}\text { El desarrollo y operación de infraestructura de redes } \\
\text { habilitadoras, fundamentales para los servicios de telecomunicaciones y } \\
\text { servicios de redes de valor añadido. }\end{array}$ \\
\hline Servicios & Servicios profesionales que no están cubiertos en otras \\
& categorías, tales como consultoría, capacitación y servicios técnicos. \\
\hline procesamiento back-office y digitalización.
\end{tabular}

Fuente: Arciniega, Flores, y García (2014)

Basándose en la información del recuadro y en busca de cifras que reflejen el sector, Dicomtech pertenecería al sector de Software (comercialización de soluciones tecnológicas de ManageEngine) y servicios (Consultoría, instalación y mantenimiento técnico) por lo que sería parte de la industria de software en el Perú. Esta conclusión llega a partir de la carencia de información sobre VARs y en búsqueda de plasmar cifras que permitan una mejor comprensión del entorno TI en el mercado peruano y la desatención del gobierno por investigarlo más a profundidad. 
Un estudio del 2003 realizado por PROMPEX y APESOFT (Asociación Peruana de Productores de Software) con el fin de determinar el potencial exportador de las empresas de software y servicios informáticos, mostraba a una industria peruana de software muy joven, pero con gran potencial de crecimiento. Las ventas totales estimadas para el año 2003 estarían por 69 millones con una tasa de crecimiento de 30 por ciento respecto al último año y con un mercado de empresas compuesto por más de 150 compañías peruanas contando solo las asociadas a APESOFT (PROMPEX Perú y APESOFT, 2003).

Posteriormente en el 2004 se presentó la Ley $N^{\circ}$ 5297, que declararía de necesidad pública e intereses nacional el desarrollo de las tecnologías de información y las comunicaciones. Otras acciones que sirvieron y sirven como impulso para el desarrollo de la industria del software son: la celebración del congreso empresarial del software que impartiría una conferencia anual, Programa de Apoyo a la Competitividad de la Industria del Software (PACIS) (Santos Hernández, 2009), Programa Crea Software Perú por PROMPERU y la más reciente siendo el cambio de nombre de cartera del Ministerio de Transportes y Comunicaciones (MTC) a Ministerio de transportes y Tecnologías de la Información y la Comunicación (MTTIC) respondiendo a la necesidad de modernizar e ir acorde a los nuevos cambios tecnológicos (Alarcón, 2018).

\subsection{Descripción de la empresa en cuanto a los accionistas, estructura organizacional, unidades de negocios, marcas y mezcla de productos}

\subsubsection{Accionistas}

Dicomtech cuenta con 2 accionistas: Luis Samanamud, dueño y Gerente General y Álvaro Samanamud, hijo del dueño. Ambos se reparten las acciones en un porcentaje que se definió en los inicios del negocio. Esto responde a la naturaleza de la empresa como S.A.C (Sociedad Anónima Cerrada) que se caracteriza entre otras cosas por ser una alternativa ideal para empresas familiares donde se permite tener un mínimo de 2 accionistas, se impone el derecho de adquisición preferente por los socios y es una persona jurídica de Responsabilidad Limitada, es decir que los socios y accionistas no responden personalmente con su patrimonio, sino con el patrimonio de la empresa. 


\subsubsection{Estructura organizacional}

Debido a que Dicomtech es una MYPE, puesto que no cuenta con más de 10 empleados y su facturación anual es menor a 150 UITs su estructura organizacional es corta y sumamente horizontal como se presenta en el siguiente gráfico:

Figura 1.2.1

Estructura organizacional de Dicomtech

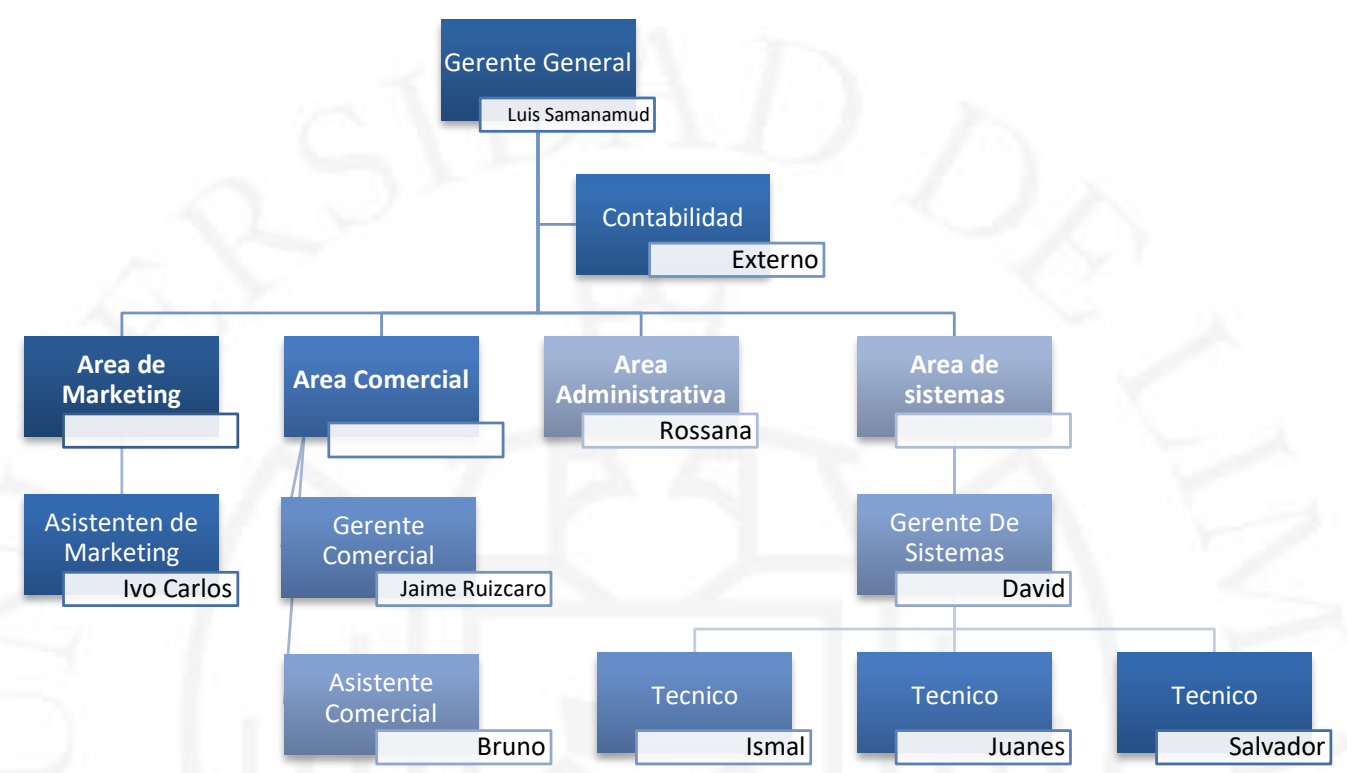

Fuente: Dicomtech (Comunicación personal, 2018)

La estructura organizacional de Dicomtech responde a un diseño organizacional común llamado estructura funcional, que se caracteriza por agrupar especialidades ocupacionales similares o relacionadas (Robbins y Coulter, 2005). Este tipo de organización permite una mejor coordinación con las áreas funcionales, y mayor eficiencia para reunir especialidades similares y personal con destreza y conocimiento, pero tiene una comunicación deficiente y una visión limitada de los objetivos organizacionales (Robbins y Coulter, 2005). 
La amplitud de control, es decir, el número de empleados que un gerente puede dirigir, es corta, dado que solo cuenta con 3 niveles y una amplitud de 4 . No requiere mayor análisis debido a la naturaleza de la compañía como MYPE. Otras características de la organización es la centralización, entendida como el grado en el que las decisiones claves de la organización son concentradas en un solo punto con... una participación escasa o nula de los niveles inferiores (Robbins y Coulter, 2005, p. 239). Dicomtech centraliza las decisiones debido a que el contacto debe ser con altos cargos de distintas compañías y que en un contexto de negocio no significa tarea para los niveles inferiores.

Es de esperar que no haya una formalización tan alta pues hay una comunicación directa con todos los niveles. Dicomtech responde a una formalización baja, donde "los comportamientos de trabajo son relativamente poco estructurados y los empleados tienen mucha libertad para realizar su trabajo" (Robbins y Coulter, 2005, p. 240). Esto último permite al empleado desempeñarse con mayor holgura, pero con un mayor peso en sus decisiones dado que estas afectaran en mayor medida el curso de la compañía.

\subsubsection{Marca y mezcla de productos}

El principal fuerte de Dicomtech es la comercialización de soluciones informáticas ManageEngine, seguido por los servicios de consultoría y un nuevo software de monitoreo y digitalización agraria llamado CropIn, con el que se está llevando a cabo acciones para su comercialización en el Perú pues la empresa Dicomtech lo representa. Anteriormente se comercializaba soluciones de seguridad BlackBerry, pero el proyecto se descontinuo debido al poco apoyo del fabricante. A continuación, se muestra la cartera de productos ME que comercializa Dicomtech. 
Tabla 1.2.1

Cartera de productos ManageEngine

\begin{tabular}{|c|c|}
\hline \multicolumn{2}{|c|}{ Productos ManageEngine } \\
\hline Categoria & Producto \\
\hline \multirow{9}{*}{ Active Directory } & AD360 \\
\hline & ADSelfService Plus \\
\hline & O365 Manager Plus \\
\hline & ADAudit Plus \\
\hline & DataSecurity Plus \\
\hline & RecoveryManager Plus \\
\hline & ADManager Plus \\
\hline & Exchange Reporter Plus \\
\hline & SharePoint Manager Plus \\
\hline Analítica & Analytics Plus \\
\hline Aplicaciones & Applications Manager \\
\hline \multirow{2}{*}{ Cloud } & AlarmsOne \\
\hline & ServiceDesk Plus \\
\hline \multirow{5}{*}{ Desktop y Mobile } & Desktop Central \\
\hline & Patch Connect Plus \\
\hline & Desktop Central MSP \\
\hline & Patch Manager Plus \\
\hline & Mobile Device Manager Plus \\
\hline \multirow{5}{*}{ Help Desk } & AlarmsOne \\
\hline & ServiceDesk Plus MSP \\
\hline & AssetExplorer \\
\hline & SupportCenter Plus \\
\hline & ServiceDesk Plus \\
\hline \multirow{6}{*}{ Seguridad de TI } & ADAudit Plus \\
\hline & Firewall Analyzer \\
\hline & Cloud Security Plus \\
\hline & Key Manager Plus \\
\hline & EventLog Analyzer \\
\hline & Password Manager Pro \\
\hline \multirow{3}{*}{ Redes y Servidores } & OpManager \\
\hline & Netflow Analyzer \\
\hline & Netword Configuration Manager \\
\hline
\end{tabular}

Fuente: ManageEngine (2018) 
El cuadro muestras las distintas líneas de productos que configuro ManageEngine porque comparten funcionalidades y características que pueden ser explicadas de forma similar. En cuanto a la extensión de línea, se puede decir que algunas categorías son cortas puesto que solo guardan dos o menos productos en su haber, sin embargo, no se puede asegurar a ciencia cierta. Hasta el momento se sabe que ManageEngine sigue agregando artículos a sus líneas de producto como lo muestra la siguiente imagen.

Figura 1.2.2

Ejemplo de lanzamiento de producto

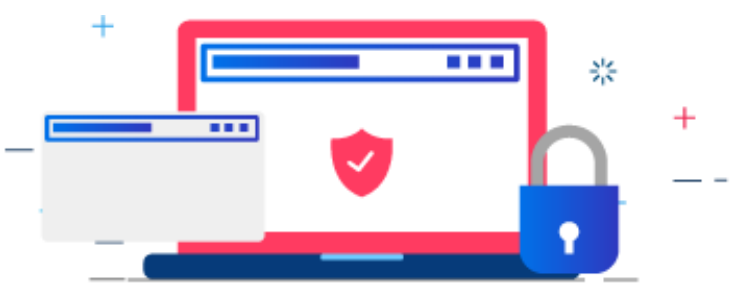

\section{ManageEngine strengthens endpoint security with the launch of Browser Security Plus}

Browser Security Plus is a browser management solution that helps organizations secure their corporate data in the cloud and protect their networks from web-based cyberattacks.

\section{Try it for free}

Fuente: ManageEngine newsletter octubre (2018)

Como se mostró en el cuadro anterior, ManageEngine cuenta con una mezcla de producto, entendida como "el conjunto de todas las líneas de productos y artículos que una determinada compañía ofrece a la venta" (Kotler y Armstrong, 2007, p. 251). Según Kotler y Armstrong (2007) "la mezcla de productos de una empresa tiene cuatro dimensiones fundamentales: ancho, extensión, profundidad y consistencia” (p. 252). 
Refiriéndose al ancho de la mezcla como el número de líneas de productos distintas que posee una compañía (Kotler y Armstrong, 2007), ManageEngine cuenta con 8 líneas de productos. En cuanto a la extensión de la mezcla de producto, es decir, "el número total de artículos que la compañía tiene dentro de sus líneas de productos” (Kotler y Armstrong, 2007, p. 252), es de 38 a más. La profundidad de la línea de productos de ManageEngine; "cantidad de versiones que se ofrecen de cada producto de la línea" (Kotler y Armstrong, 2007, p. 252) suele ser de 2 (Essential y Enterprise), y en ocasiones de 3 cuando existe una versión plus como lo muestra el ejemplo a continuación sobre versiones de OpManager.

Figura 1.2.3

Ejemplo de versiones producto OpManager

\begin{tabular}{|c|c|c|c|}
\hline & $\begin{array}{c}\text { Essential } \\
\text { Para pequeñas y medianas empresas }\end{array}$ & $\begin{array}{l}\text { OpManager Plus } \\
\text { Paquete de licencia especial }\end{array}$ & $\begin{array}{c}\text { Enterprise } \\
\text { Para grandes empresas }\end{array}$ \\
\hline Monitoreo de redes distribuidas & $x$ & $x$ & $\checkmark$ \\
\hline Mapa de red L2 automático & $\checkmark$ & $\checkmark$ & $\checkmark$ \\
\hline $\begin{array}{l}\text { Monitoreo básico [Windows/ Linux/ SQL/ AD/ } \\
\text { mySQL/ eventlog/ Trap/ SNMP }\end{array}$ & $\checkmark$ & $\checkmark$ & $\checkmark$ \\
\hline $\begin{array}{l}\text { Monitoreo avanzado [Vmware/ Hyper-V/ Xen } \\
\text { Monitoring/ UCS] }\end{array}$ & $\checkmark$ & $\checkmark$ & $\checkmark$ \\
\hline Add-On's & $\begin{array}{l}\text { NFA [Gratis para } 2 \text { interfaces] } \\
\text { NCM [Gratis para } 2 \text { dispositivos] } \\
\text { IPAM [Gratis para } 50 \text { IPs \& puertos de } \\
\text { switch utilizados] } \\
\text { Failover } \\
\text { Monitoreo de WAN RTT } \\
\text { Monitoreo de VoIP } \\
\text { Motor de alto rendimiento } \\
\text { ASAM } \\
\text { Monitoreo DPI }\end{array}$ & $\begin{array}{l}\text { NFA [Interfaces }]^{*} \\
\text { NCM [Dispositivos] }{ }^{*} \\
\text { Firewall [Dispositivos] }]^{*} \\
\text { IPAM [IPs \& puertos de switch utilizados] } \\
\text { Failover } \\
\text { Monitoreo de WAN RTT } \\
\text { Monitoreo de VolP } \\
\text { Motor de alto rendimiento } \\
\text { ASAM } \\
\text { Monitoreo DPI }\end{array}$ & $\begin{array}{l}\text { NFA [Gratis para } 2 \text { interfaces] } \\
\text { NCM [Gratis para } 2 \text { dispositivos] } \\
\text { IPAM [Gratis para } 50 \text { IPs \& puertos de } \\
\text { switch utilizados] } \\
\text { Monitoreo de WAN RTT } \\
\text { Monitoreo de VolP } \\
\text { Motor de alto rendimiento } \\
\text { ASAM } \\
\text { Monitoreo DPI }\end{array}$ \\
\hline Plug-In's & Plug-in APM [5 monitores por defecto] & Plug-in APM [5 monitores por defecto] & Plug-in APM [5 monitores por defecto] \\
\hline Cantidad máxima de dispositivos & 1000 & 1000 & 50000 \\
\hline
\end{tabular}

Fuente: ManageEngine (2018) 
Esto se debe a que cada versión es mejor que la anterior, tiene costos diferentes, características aumentadas y está a disposición de los requerimientos de los clientes. Finalmente, en cuanto a la consistencia de la mezcla de productos de ManageEngine, entendida como el relacionamiento de las distintas líneas de productos respecto a su uso final, necesidades, canales, entre otros (Kotler y Armstrong, 2007) es alta, o muy consistente pues usan los mismos canales de distribución, están dirigidas a medianas/ grandes empresas, son software de administración de TI y usan dispositivos similares, entre otros aspectos.

\subsection{Descripción del mercado y sus segmentos}

Según el Instituto Nacional de Estadística e Informática (INEI) las empresas que se consideran mediana y grandes empresas deben tener ventas anuales mayores a 1700 y 2300 UIT respectivamente considerando que el límite superior de la mediana empresa es de 2300 UIT (INEI, 2015). Según el diario El Peruano un UIT en 2018 esta valorizado en 4,150 soles (Decreto Supremo N 380-2017-EF, 2018).

El Directorio Central de empresas y Establecimientos elaborado por el INEI, registra hasta el 31 de diciembre de 2016, 2 millones 124 mil 280 empresas que desarrollaron actividades económicas en el territorio nacional. Esta información proviene de la Superintendencia Nacional de Aduanas y de Administración Tributaria (SUNAT) y de las encuestas de empresas del Sistema Estadístico Nacional (SEN) (INEI, 2016)

Figura 1.3.1

Perú: Empresas, según segmento empresarial, 2015-16

\begin{tabular}{|c|c|c|c|c|}
\hline \multirow{2}{*}{ Segmento empresarial } & \multirow{2}{*}{2015} & \multicolumn{2}{|c|}{2016} & \multirow{2}{*}{$\begin{array}{r}\text { Var \% } \\
2016 / 15\end{array}$} \\
\hline & & Absoluto & Porcentaje & \\
\hline Total & 2042992 & 2124280 & 100,0 & 4,0 \\
\hline Microempresa & 1933525 & 2011153 & 94,7 & 4,0 \\
\hline Pequeña empresa & 89993 & 92789 & 4,4 & 3,1 \\
\hline Gran y mediana empresa & 12494 & 13031 & 0,6 & 4,3 \\
\hline Administración pública & 6980 & 7307 & 0,3 & 4,7 \\
\hline
\end{tabular}

Fuente: INEI (2016) 
Basándose en el anterior cuadro, se puede concluir que el mercado al que apunta Dicomtech está compuesto por la gran y mediana empresa y la administración pública que da un total de 20,338 empresas que equivale a 0,9 por ciento del segmento empresarial. Esto sin tomar en cuenta otras variables de suma importancia como lo son: número de técnicos en la compañía, dispositivos, redes, usuarios, entre otros que reducirían aún más este mercado.

\subsection{Cuantificación de la industria en volumen de unidades y/o valor de la venta}

La industria tecnológica en el Perú cerró el 2016 en 3,887 millones de dólares, pero el sector software del cual es parte Dicomtech se prevé que crezca de manera constante pues el año 2016 cerró en 240 millones de dólares y se proyecta un crecimiento de 5,3 por ciento en el 2017 según Carlos Jacobsen, gerente general de la Asociación Peruana de Software y Tecnologías (Apesoft). (“Apesoft: Con entidad dedicada a las TIC se duplicaría el crecimiento del sector software", 2017)

Además, El ejecutivo planteó que el ratio podría subir a 12 por ciento, siempre y cuando se implemente el Ministerio de Tecnologías de Información y Comunicación, que como se explicó líneas arriba, ya es una realidad conformado por el MTC (Ministerio de transporte y Comunicaciones). Actualmente existen 500 empresas peruanas de software, pero solo 95 pertenecen a Apesoft. Aun así, estas representan el 80 por ciento de la facturación del mercado según Jacobsen. Se debe recalcar que el crecimiento de la industria viene acompañado de las acciones de Apesoft como la conformación de una mesa de trabajo con el MTC para plantear los cimientos del Viceministerio de las TIC.

Otro dato importante es la falta de información del sector de Tecnologías de Información en Perú, en palabras del Gerente general de Apesoft "El estado como que nos ha abandonado... a pesar de que tenemos un instituto nacional de estadística, no ha visualizado la necesidad del sector de tener información que nosotros podamos usar" (Apesoft: Con entidad dedicada a las TIC se duplicaría el crecimiento del sector software, 2017) 


\subsection{Identificación, descripción y participación de mercado de competidores directos}

La identificación de los principales competidores se extrae de la base de datos recientemente adquirida por Dicomtech complementándose con el Estudio de Mercado Servicio Industria del Software en Perú elaborado por la oficina comercial de chile (ProChile, 2013) y que a su vez comenta en el documento que:

"Dado que no existen estadísticas oficiales que permitan hacer seguimiento con precisión de la industria del software en el Perú, en términos de Producto Bruto Interno (PBI) sectorial, ventas nacionales, exportaciones, empleo e inversión, se analizará de manera de tener un referente, el mercado de Tecnologías de la Información y Comunicaciones (TIC)” (p. 6).

Basándose en esto último las principales empresas competidoras identificadas en la base de datos tomando en cuenta las 10 primeras por razón social según el ranking de facturación son: IBM DEL PERU, GMD, SISTEMAS ORACLE DEL PERU, SAP PERU, SALESLAND INTERNACIONAL, PC LINK, MOVILWAY PERU, NEXUS TECHNOLOGY, SOFTWAREONE PERU, AFINA PERU. Cuentan con ventaja competitiva, recursos, volúmenes de venta, ubicación, experiencia en el mercado, procesos, productos, canales, medios de publicidad.

Los servicios de estas empresas están vinculados al desarrollo de diversas herramientas que van desde software para procesos de ingeniería, educativos, call center, hasta aplicaciones App para el monitoreo de redes de celulares interna o externa. Estas aplicaciones tecnológicas para la gestión del negocio son dirigidas al manejo de grandes volúmenes de información (ProChile, 2013). Es por ello que DICOMTECH busca y vende a medianas y grandes empresas pues empresas de menor envergadura no entran dentro de la categoría puesto que no vale la pena venderles una licencia.

El siguiente cuadro muestra las 20 primeras empresas según el ranking de facturación extraída de las bases de datos adquiridas, los números son estimados por lo que se muestra una facturación mínima y máxima. Se debe tomar en cuenta además que la facturación a partir de una posición dentro del ranking no se encuentra por lo que existe falta de datos. Por último, se constató con otra base de datos que el subsector al que pertenecen estas empresas es el de Sistemas: producción de software / software consultancy, y detalla que las empresas son tanto consultores en programas de informática como suministradores. 
Tabla 1.5.1

Ranking de empresas por facturación

\begin{tabular}{|c|c|c|c|c|}
\hline Razón Social & Sector Esp & Sector Ing & $\begin{array}{l}\text { Facturado } 2016 \\
\text { Soles Mínimo }\end{array}$ & $\begin{array}{l}\text { Facturado } 2016 \text { Soles } \\
\text { Máximo }\end{array}$ \\
\hline IBM DEL PERU S.A.C. & INFORMATICA & COMPUTATION & $1,040,000,000.00$ & $1,229,999,999.00$ \\
\hline GMD S.A. & INFORMATICA & COMPUTATION & $216,000,000.00$ & $237,999,999.00$ \\
\hline SISTEMAS ORACLE DEL PERU S.A. & INFORMATICA & COMPUTATION & $170,000,000.00$ & $189,999,999.00$ \\
\hline SAP PERU S.A.C. & INFORMATICA & COMPUTATION & $160,000,000.00$ & $169,999,999.00$ \\
\hline SALESLAND INTERNACIONAL S.A. & INFORMATICA & COMPUTATION & $118,000,000.00$ & $130,999,999.00$ \\
\hline PC LINK S.A.C. & INFORMATICA & COMPUTATION & $109,000,000.00$ & $117,999,999.00$ \\
\hline MOVILWAY PERU S.A.C. & INFORMATICA & COMPUTATION & $77,000,000.00$ & $91,999,999.00$ \\
\hline NEXUS TECHNOLOGY S.A.C. & INFORMATICA & COMPUTATION & $77,000,000.00$ & $91,999,999.00$ \\
\hline SOFTWAREONE PERU S.A.C. & INFORMATICA & COMPUTATION & $77,000,000.00$ & $91,999,999.00$ \\
\hline AFINA PERU S.A.C. & INFORMATICA & COMPUTATION & $77,000,000.00$ & $91,999,999.00$ \\
\hline MICROSOFT PERU S.R.L. & INFORMATICA & COMPUTATION & $63,600,000.00$ & $76,999,999.00$ \\
\hline GRUPO SYPSA S.A.C. & INFORMATICA & COMPUTATION & $63,600,000.00$ & $76,999,999.00$ \\
\hline $\begin{array}{l}\text { TATA CONSULTANCY SERVICES SUC. DEL } \\
\text { PERU }\end{array}$ & INFORMATICA & COMPUTATION & $63,600,000.00$ & $76,999,999.00$ \\
\hline ROCKWELL AUTOMATION DE PERU S.A. & INFORMATICA & COMPUTATION & $54,500,000.00$ & $63,599,999.00$ \\
\hline CIMACOM DATA S.A.C. & INFORMATICA & COMPUTATION & $46,000,000.00$ & $54,499,999.00$ \\
\hline SEIDOR CONSULTING PERU S.A.C. & INFORMATICA & COMPUTATION & $46,000,000.00$ & $54,499,999.00$ \\
\hline COMPUSOFT S.R.L. & INFORMATICA & COMPUTATION & $42,500,000.00$ & $45,999,999.00$ \\
\hline TECHNOLOGY OUTSOURCING S.A.C. & INFORMATICA & COMPUTATION & $42,500,000.00$ & $45,999,999.00$ \\
\hline INNERWORKINGS PERU S.A.C. & INFORMATICA & COMPUTATION & $42,500,000.00$ & $45,999,999.00$ \\
\hline
\end{tabular}

Fuente: Base de datos TOP 100,000.

Si bien en líneas anteriores se mencionó una cifra respecto al sector software específicamente, se asume que no todas las empresas del siguiente ranking pertenecen a Apesoft y que este maneja información limitada del mercado, así como el público en general

En cuanto al Software CropIn, dirigido a los agro industriales para el monitoreo y digitalización de sus fundos, el competidor más arraigado al sector Agri-Tech es SAP que desde hace 10 años viene comercializando su software para Fundos de gran tamaño y que están consolidados por una empresa. 


\section{CAPÍTULO II: DIAGNÓSTICO DEL MARKETING ESTRATÉGICO DE LA EMPRESA}

\subsection{Descripción y análisis de la estrategia genérica}

Es necesario destacar a Michael Porter, quien en distintas publicaciones ha señalado las diferentes estrategias que se pueden utilizar para conseguir eficazmente una ventaja competitiva (Porter, 2008). Las resume en 3 categorías:

Liderazgo en costos: se refiere a llevar a cabo un conjunto de políticas funcionales encaminadas al objetivo de controlar los costos y mantenerlos bajos frente a la competencia sin descuidar la calidad, el servicio ni otros aspectos. (p. 52)

Diferenciación: requiere que el producto o servicio ofrecido al consumidor deba ser percibido como algo único en el sector y a la vez difícil de igualar para evitar a la competencia. (p. 54)

Enfoque: concentrar los esfuerzos en un grupo de compradores, en un segmento de la línea de productos o en un mercado geográfico, previamente analizando y eligiendo el mercado bajo la suposición de que la compañía podrá prestar una mejor atención a su segmento que las empresas que compiten en mercados más extensos. (p. 55)

Habiendo mencionado esto, Dicomtech utiliza una estrategia de liderazgo en costos pues busca vigorosamente la reducción de costos a partir de la experiencia, controla los gastos variables y fijos, evita clientes pequeños y minimiza costos en áreas como marketing e investigación. Esto además de ser representante de los productos de ManageEngine, cuya naturaleza es de costos muy por debajo de la industria nacional y que le ha permitido consolidar una cartera de clientes conformada por empresas de gran extensión y recorrido. 


\subsection{Descripción y análisis de la(s) ventaja(s) competitiva(s)}

De acuerdo a Ferret y Hartline (2012), "una ventaja competitiva es algo que la empresa hace mejor que sus competidores, que le otorga superioridad para satisfacer las necesidades de los clientes o para mantener relaciones mutuamente satisfactorias con los grupos de interés importantes" (p. 18). Se debe recordar que las ventajas derivan de las fortalezas que la empresa posee, en este caso Dicomtech destaca por el equipo de técnicos especializados que posee y que cumple con una de las ocho fuentes comunes de ventaja competitiva mencionada en el texto de Ferret y Hartline (2012).

La ventaja de recursos humanos está basada en el destaque de algún factor del elemento humano de la compañía sea o no de alta dirección. En el caso de Dicomtech, cuenta con acceso a mano de obra calificada pues las personas que conforman el staff técnico cuentan con estudios superiores y alto conocimiento de los productos que venden; son empleados comprometidos, ya que comparten la misión y visión de la empresa y tienen capacitación de clase mundial, el principal miembro del equipo fue capacitado en la india. Matriz de ManageEngine.

\subsection{Descripción y análisis de la(s) estrategia(s) de marketing corporativo que aplica}

La estrategia corporativa establece principalmente el rumbo de una empresa en general y la administración de su cartera de negocios o productos, ya sea pequeña o una corporación multinacional grande, como ManageEngine. Wheelen y Hunger (2007) mencionan que la estrategia corporativa se ocupa de tres aspectos clave que enfrente la corporación en general:

1. La orientación general de la empresa hacia el crecimiento, la estabilidad o la reducción (estrategia direccional).

2. Las industrias o mercados en los que compite la empresa por medio de sus productos y unidades de negocio (estrategia de cartera).

3. La manera en que la administración coordina las actividades, transfiere los recursos entre las líneas de productos y cultiva las capacidades de negocio. 
Con los aspectos antes mencionados, se puede determinar en resumen que Dicomtech no lleva a cabo ningún tipo de estrategia corporativa pues su posición de comercializador no se lo permite y no le concierne tampoco como partner, pues la misma asociación no le quita autonomía a Dicomtech. Los encargados de llevar a cabo estas acciones son ManageEngine y CropIn, pues son los fabricantes y dueños de las patentes del software que comercializan.

\subsection{Descripción y análisis de la(s) estrategia(s) competitiva(s) de marketing que aplica}

Kotler y Keller (2016) detallan 4 estrategias competitivas que se pueden utilizar para hacer frente a la competencia:

Líder: empresa que maneja el mayor SOM en la categoría donde se desarrolla. Se enfoca principalmente en reforzar y mantener su posición. Dentro de las estrategias para esta posición se encuentran: Expansión de la demanda total de mercado, Protección de la participación de mercado e incremento de la participación de mercado (p. 337-341)

Retador: empresa que le sigue al líder en lo que SOM respecta. Tiene el objetivo de acercarse y superar al líder en el corto/mediano plazo, al igual que evitar que los seguidores no le quiten participación. Destaca principalmente por que las estrategias de la posición están enfocadas al ataque (p. 343-344)

Seguidor: mayoría de empresas presentes en el sector. Preferencia por seguir al líder en vez de retarlo debido a que la posibilidad de diferenciación de producto y de imagen son escasas, se requiere mucha inversión o hay mucha sensibilidad de precio. Las estrategias para esta posición son la de Clonador, Imitador y Adaptador. (p. 344)

Especialista: empresa que busca ser líder en un mercado pequeño o de nicho, evitando la confrontación directa con las grandes empresas, dirigiéndose a mercados más limitados que no son de interés para las grandes organizaciones. Kotler plantea que los especialistas deben ejecutar tareas, crear nichos, expandirlos y protegerlos. (p. 346-347)

Dicomtech no sigue una estrategia competitiva de marketing puesto que se basa en las acciones que ManageEngine toma a cabo. A pesar de ello, se trata de trazar constantemente las acciones de la competencia. 


\subsection{Descripción del(los) grupo(s) objetivo que atiende}

Kotler (2007) señala que: "un mercado meta consiste en un conjunto de compradores que tienen necesidades o características comunes, y a los que la compañía decide servir" (p. 178). En este marco, para visualizar mejor las características del público objetivo de Dicomtech, se utilizara la segmentación denominada "enfoque de nidos" de Shapiro y Bonoma (1984), en base a valoraciones del dueño de la compañía y observaciones.

Figura 2.5.1

Enfoque de Nido

\section{Enfoque de Nido}

Factores Demográlicos

Variables 0peratimas

Enfoque de Compras

Factores Situacionales

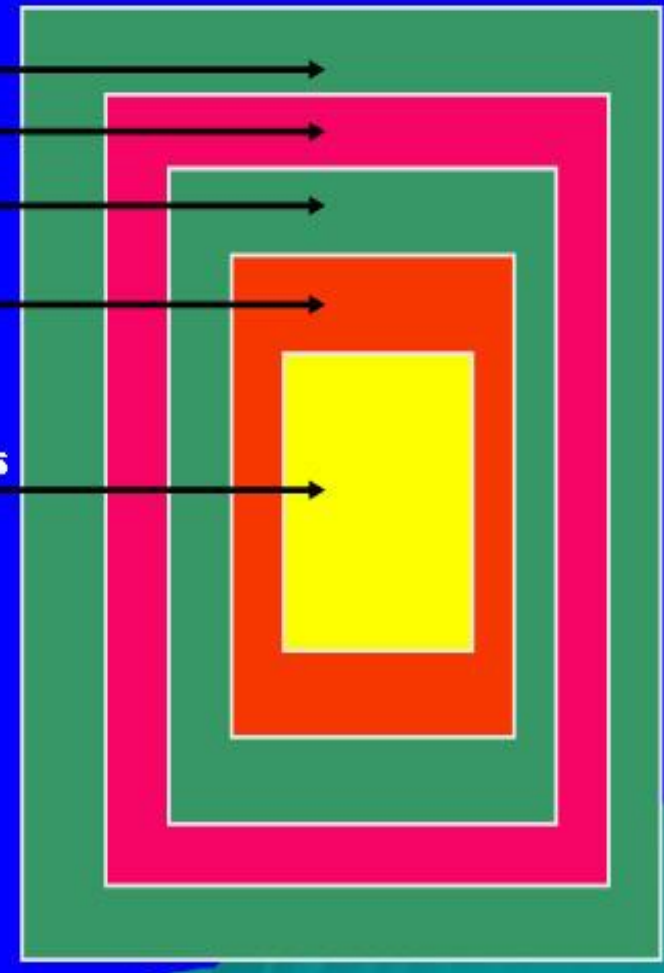

Fuente: Bonoma y Shapiro

Fuente: Shapiro y Bonoma (1984) 
La siguiente tabla muestra la segmentación del público objetivo realizada en base a los criterios del enfoque de nido.

Tabla 2.5.1

Segmentación del público objetivo

\begin{tabular}{|c|c|}
\hline Sector & Descripción \\
\hline Bienes & $\begin{array}{l}\text { Producción de bienes de consumo de T.I. tales como equipos } \\
\text { informáticos y de telecomunicaciones digitales, bienes de capital (por } \\
\text { ejemplo, maquinaria automatizada para la fabricación de Computadoras } \\
\text { Personales) y bienes intermedios tales como placas base, discos duros, } \\
\text { unidades de DVD, entre otros periféricos, utilizados en la fabricación de } \\
\text { computadoras. }\end{array}$ \\
\hline Software & $\begin{array}{l}\text { Diseño, producción, mantenimiento y comercialización de } \\
\text { software empaquetado y/o personalizado. }\end{array}$ \\
\hline Infraestructura & $\begin{array}{l}\text { El desarrollo y operación de infraestructura de redes } \\
\text { habilitadoras, fundamentales para los servicios de telecomunicaciones y } \\
\text { servicios de redes de valor añadido. }\end{array}$ \\
\hline Servicios & $\begin{array}{l}\text { Servicios profesionales que no están cubiertos en otras } \\
\text { categorías, tales como consultoría, capacitación y servicios técnicos. }\end{array}$ \\
\hline Contenido & $\begin{array}{l}\text { Producción y distribución de contenido de datos, incluyendo el } \\
\text { procesamiento back-office y digitalización. }\end{array}$ \\
\hline
\end{tabular}

Fuente: Elaboración propia

\subsection{Descripción del posicionamiento de la empresa o marca(s)}

Kotler y Armstrong (2007) detallan la posición de un producto como la forma en que los consumidores definen el producto en base a sus atributos importantes, también entendido como el lugar que el producto ocupa en la mente del consumidor respecto a los productos de la competencia. 
El posicionamiento de Dicomtech se basa en la asociación o mejor conocida como 'partnership' que estableció con ManageEngine a sus inicios para fungir de representante de la marca desarrolladora de software en el país. Esto no quiere decir que ManageEngine no cuenta con otros representantes o comercializadores en el país, pero Dicomtech formaría una de las principales fuerzas de venta de ManageEngine, así como socio al compartir gastos en la realización de eventos, seminarios, talleres, entre otros.

El segundo beneficio que ostenta Dicomtech es el bajo precio, que si bien viene a raíz del bajo costo puede y es modificado por los comercializadores locales donde incluyen el margen y el costo del servicio. Este último punto completaría la última arista del posicionamiento de Dicomtech, destacándose por el valor de su staff técnico que cuenta con capacidad, conocimientos y visión compartida. Son estos tres atributos los que componen la propuesta de valor, la mezcla completa de beneficios con base a las cuales se posiciona.

Si nos ubicamos dentro del cuadro de posibles propuesta de valor desarrollado por Kotler y Armstrong (2007), Dicomtech se encontraría en el cuadrante llamado "Más por Menos", dado que ofrece un producto y servicio de alta calidad a un precio bajo respecto a la industria, recordando que los competidores varían según el producto que ofrezcan, también se podría encontrar en el cuadrante "Lo mismo por menos", posicionamiento que sigue confiriendo a la compañía una ventaja competitiva. Esto se refleja en los bajos márgenes de ganancia que a veces maneja la compañía y que dificulta el mantenimiento de la posición a largo plazo

Tabla 2.6.1

Posibles Propuestas de valor

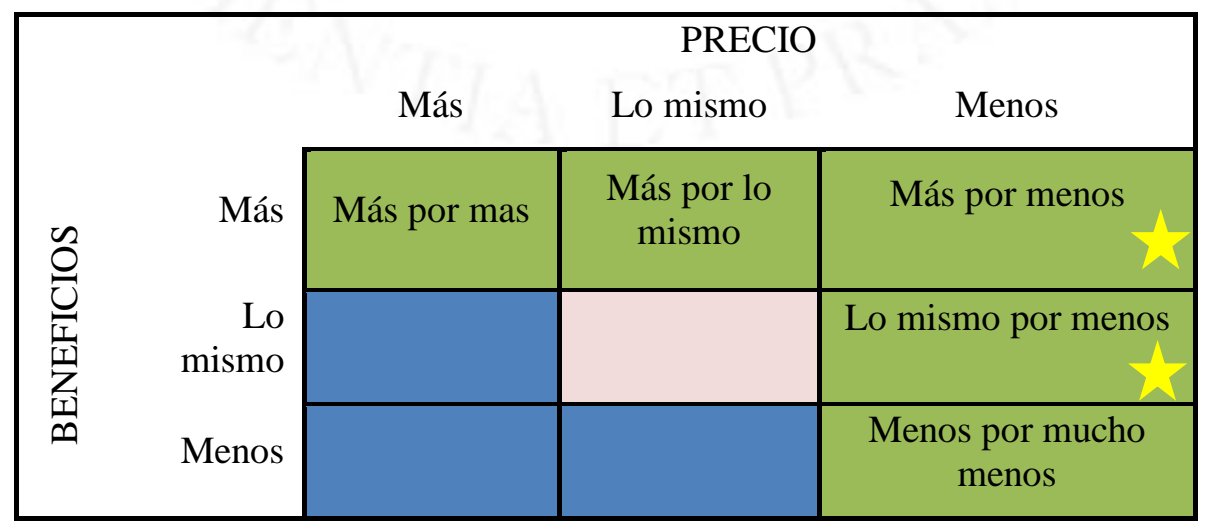

Fuente: Kotler y Armstrong (2007) 


\subsection{Descripción de la creación del Brand Equity de su(s) marca(s)}

Según Kotler y Keller (2012)

Los especialistas en marketing construyen Brand Equity mediante la creación de estructura de conocimiento de marca apropiadas en los consumidores adecuados. Este proceso depende de todos los contactos que se establecen con la marca, ya sea que los inicie o no el especialista en marketing. (p. 249).

Se evaluará el Brand Equity de Dicomtech tomando en cuenta la marca en sí y las acciones de marketing que lleva a cabo la compañía. En ese sentido, Kotler y Keller (2012) plantean desde la perspectiva de la dirección de marketing tres conjuntos de factores que impulsan el Brand Equity:

a) La elección inicial de los elementos o identidades que conforman la marca (nombre de marca, URL, logotipo, símbolos, personajes, portavoces, slogan, jingles, signos, entre otros). (p. 249)

b) El producto o servicio y todas las actividades de marketing y los programas y refuerzos relacionados. (p. 249)

c) Otras asociaciones transferidas indirectamente a la marca, mediante su vinculación con otra entidad. (p. 250)

Sobre el primer conjunto de factores, el nombre de marca de Dicomtech es una abreviatura de Digital Communication Technology. Como menciona Keller (2008), El nombre de marca puede ser un medio de comunicación abreviado extremadamente eficaz, además se cumple con la simplicidad y la fácil pronunciación, es diferenciado, distintivo y único y hace alusión a la tecnología, rubro donde se desempeña Dicomtech.

La URL de Dicomtech es www.dicomtech.com.pe y se encuentra debidamente registrada para evitar su suplantación. El logotipo cae en el extremo de ser una representación literal del nombre pero que logra una asociación con la tecnología por los acabados con los que cuenta. 
Respecto al segundo conjunto de factores, Dicomtech realiza distintas actividades de marketing para construir valor capital de marca. Dentro de estas actividades encontramos:

- Email Marketing: que engloba no solo presentaciones de producto, sino información relevante para el cliente, como guías, e-books, infografías, entre otros

- Webinars: Propios y Externos

- Eventos: Seminarios, capacitaciones y otras actividades presenciales donde se traen eminencias o personas altamente capacitadas gracias al apoyo de ManageEngine

Por último, respecto a las asociaciones que ha logrado conseguir Dicomtech, la más significativa es como Partner exclusivo del desarrollador de software transnacional ManageEngine que le ha permitido consolidarse más en el rubro en el que se desempeña.

Las siguientes imágenes muestran los anteriores factores mencionados aplicados a la empresa DICOMTECH.

Figura 2.7.1

Logo Dicomtech

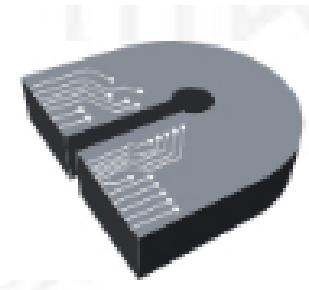

$\underset{\text { TOP it Solutions for the business }}{\text { DIOOMMTECH }}$

Fuente: Dicomtech (2018a) 
Figura 2.7.2

Campaña de email marketing

De : DICOMTECH <info@dicomtech.com.pe

Respuesta : info@dicomtech.com.pe

Asunto : Diez controles de seguridad para una ciberseguridad efectiva - Infografía

\section{DICOMTECH.}

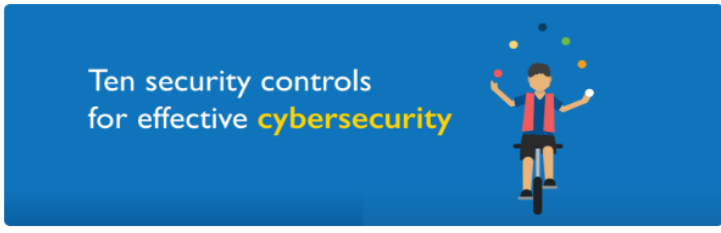

La amenaza de ciberataques continúa creciendo tanto para desktops como para

dispositivos móviles. La peor parte es que parece no tener fin. Todo lo que las

compañias pueden hacer para proteger sus redes es asegurarse de tener todos los

controles correctos preparados con el fin de prevenir estos ataques.

Esta infografia le ayudará a entender mejor estos diez controles criticos de

seguridad. También tiene información sobre el impacto que estos controles tienen en el

mercado, cómo determinar los mejores controles para su compañía y cómo implementar

todos estos controles únicos a la vez.

Descargar infografia

Fuente: Dicomtech (comunicación personal, 2018b)

Figura 2.7.3

Webinars CropIn

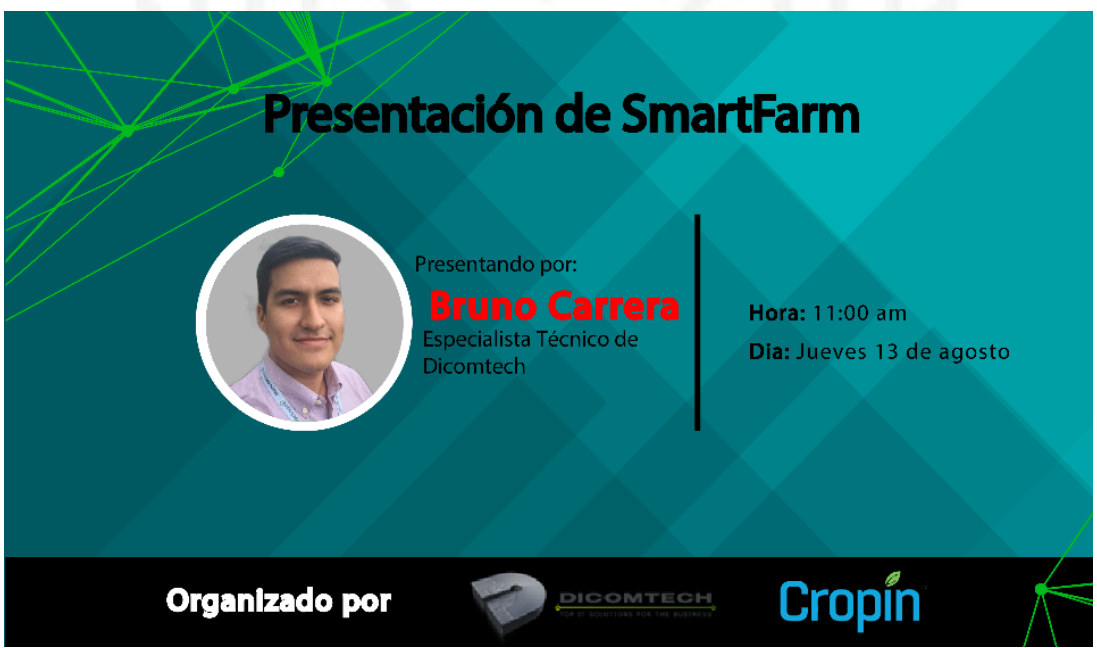

Dicomtech (comunicación personal, 2018c) 
Figura 2.7.4

Seminario de seguridad de TI y Active Directory

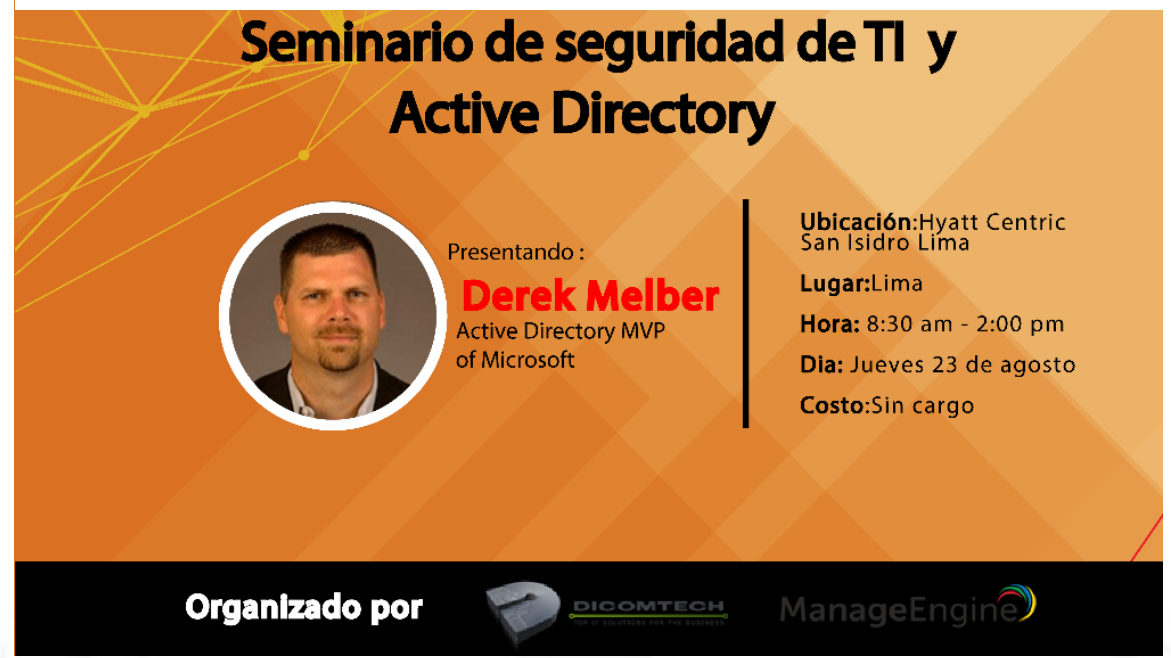

Fuente: Dicomtech (comunicación personal, 2018d) 


\section{CAPÍTULO III: DESCRIPCIÓN DEL CASO PROBLEMA}

\subsection{Descripción de la situación}

En puntos anteriores se ha hablado sobre los antecedentes, mercado, competencia, producto y presente de Dicomtech, dando así un marco general de la situación actual que enfrenta y permitiendo avanzar directamente a uno de los problemas que refiere al componente de marketing denominado Precio.

Se planteó como solución una investigación de mercado que tendrá como eje central el marco cualitativo debido al reducido universo con el que se cuenta y que hace difícil la tarea de cuantificarlo y apalancarse en sus resultados. Para dicha investigación se empezará describiendo el problema, planteando los objetivos y posteriormente brindando soluciones que busquen resolver el problema de imagen con el que cuenta Dicomtech.

En los años que tiene Dicomtech como comercializador de ME, el dueño se ha percatado que el precio establecido está muy por debajo de la competencia, considerando que esta difiere respecto a la naturaleza del producto (Administración de Service Desk, Logs Outs, Active Directory, etc.) y que además los clientes valoran el servicio que viene acompañado por el producto (licencia de software) como mantenimiento, reposición, instalación, ayuda técnica, etc.

Es la valoración del cliente por el servicio lo que pone en tela de juicio el precio establecido, teniendo como segundo lugar el precio de la competencia. Los precios que se manejan respecto a la competencia que no son VARs, es decir, los desarrolladores de software, son más bajos como se muestra a continuación en la comparación de un producto de gestión de redes similar a OpManager llamado Network Performance Monitor de Solarwinds, otro fabricante de software que comercializa sus productos en el Perú.

OpManager cuenta con distintos paquetes respecto a los requerimientos del cliente siendo el más bajo el llamado "25 devices Special Pack" a un precio total, incluyendo impuestos de 858 dólares americanos. Este será el producto a comparar junto a Solarwinds. 
Figura 3.1.1

Precios de paquetes OpManager

\begin{tabular}{l|lc} 
Initial Product Fee (IPF) & Get Quote & \\
\\
Base Product & & \\
\hline Products & Initial License Fee & ASF* \\
$\square 25$ Devices Special Pack & US $\$ 715$ & US $\$ 143$ \\
$\square 50$ Devices Pack & US $\$ 2,395$ & US $\$ 479$ \\
$\square 100$ Devices Pack & US $\$ 4,195$ & US $\$ 839$ \\
$\square 250$ Devices Pack & US $\$ 6,595$ & US $\$ 1,319$ \\
$\square 500$ Devices Pack & US $\$ 9,595$ & US $\$ 1,919$ \\
$\square 1000$ Devices Pack & US $\$ 14,995$ & US $\$ 2,999$ \\
(For more than 1000 devices, buy Enterprise edition) & &
\end{tabular}

Fuente: ManageEngine (2018)

Para entender el alcance y el número de máquinas que abarca la primera licencia se hace uso de la siguiente tabla. Esta muestra un detalle de la estructura de licencia de OpManager Plus, exactamente igual a OpManager, pero con algunas características aumentadas. Lo que se rescata del siguiente cuadro es la última columna que indica el IPAM (IPs y puertos de switch utilizados); entendiéndose el IP como la matrícula de un dispositivo en la red, se puede determinar que el primer paquete "50 Pack" cubre 250 IPs, es decir, 250 computadoras.

Para determinar cuántas PCs cubre la licencia "25 devices Special Pack" del cuadro superior se hace una regla de 3 simple donde se determina que la licencia cubre 125 IPs, es decir 125 computadoras.

Figura 3.1.2

Detalles de la estructura de licencia de OpManager Plus

OpManager Plus

OpManager (Dispositivos)

NFA (Interfaces)

NCM (Dispositivos)

Firewall IPAM (IPS

(Dispositivos) y puertos

de switch

utilizados)

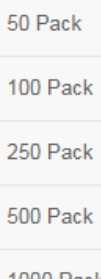

1000 Pack

\begin{tabular}{|l|l|}
\hline 50 \\
\hline 100 \\
\hline 250 & 25 \\
\hline 500 & 100 \\
\hline 1000 & 250 \\
\hline
\end{tabular}

\begin{tabular}{|c|c|c|c|}
\hline 15 & 15 & 1 & 250 \\
\hline 25 & 25 & 1 & 500 \\
\hline 50 & 50 & 1 & 1000 \\
\hline 100 & 100 & 2 & 2000 \\
\hline 250 & 250 & 2 & 4000 \\
\hline
\end{tabular}

Fuente: ManageEngine (2018) 
En cuanto al producto "Network Performance Monitor SL100” de Solarwinds, El anexo C, muestra que cubre hasta 100 elementos, entendiéndose elementos como 100 computadoras. Es decir, en una comparación final, se puede llegar a la conclusión de que por el mismo número de computadoras OpManager cuesta 2,097 dólares menos. La abismal diferencia se debe a los costos extremadamente bajos que puede manejar ManageEngine en la India.

Por último, si bien es útil la presencia de ManageEngine como recurso de venta, la marca Dicomtech se ve afectada y sobrecogida por el fabricante y por otros revendedores directos que venden casi al precio original del fabricante lo que le quita margen de contribución a Dicomtech, lo que significaría que un incremento de precio (pese a depender del revendedor) podría afectar la percepción del consumidor si esta no va acompañada de acciones de marketing, comunicacionales de imagen, entre otras. La llave para mejorar los márgenes de ventas también recae en proveer servicios de valor agregado de calidad que incrementen la satisfacción y retención de los consumidores. 


\section{CAPÍTULO IV: ANÁLISIS Y DISCUSIÓN DE RESULTADOS}

\subsection{Definición del Problema}

El problema es que la empresa no ha sabido posicionar (poner en valor) adecuadamente el servicio en cuanto al precio, ya que este solamente le genera márgenes bajos. Ahora la empresa requiere reposicionar su servicio apoyado en productos a través de una nueva imagen que ponga en evidencia los atributos y características de alto valor entre los clientes.

\subsection{Objetivo}

Averiguar cuáles son los atributos y características más valorados por los clientes para elegir a un proveedor de licencias de software (respecto a categoría y en general).

Algunos ejemplos de características o atributos pueden ser: nivel técnico, capacidad de ajustes a requerimientos de los clientes, atención, mantenimiento, capacitación, auditoria, entre otros.

\subsection{Objetivos Específicos}

- Explorar los softwares que ha usado, sus proveedores, características, motivaciones,

- Indagar en la imagen y posicionamiento de los proveedores actuales

- Profundizar en las fortalezas y debilidades de los proveedores y competidores actuales.

- Sondear en las oportunidades de mejora y sugerencias para que los proveedores actuales brinden un mejor servicio.

- Indagar en los problemas que ha tenido en el pasado con los proveedores.

- Explorar los atributos que calidad percibida (antigüedad de la empresa, respaldo de empresa internacional, entre otros). 


\subsection{Diseño de la investigación}

Según Malhotra (2008), la investigación exploratoria es un tipo de diseño de la investigación que proporciona información y comprensión del problema que enfrenta el investigador y que una de sus características es que la muestra es pequeña y no representativa y el análisis de los datos primarios es cualitativo (p. 80). Son estos dos últimos puntos los que soportan la elección de este tipo de diseño debido a la inviabilidad de un estudio cuantitativo, tomando principalmente en cuenta que la muestra sería muy minúscula debido al pequeño universo de organizaciones que entran en la categoría de mediana y gran empresa.

\subsection{Métodos a utilizar}

\subsubsection{Datos secundarios}

Como primer método se buscará recopilar datos de fuentes externas, es decir, datos secundarios, los cuales incluyen información que se obtuvo de fuentes privadas, gubernamentales, de empresas comerciales dedicadas a la investigación de mercados o de bases de datos. (Malhotra, 2008). Para tal caso, se buscará realizar una revisión de literatura académica y comercial para identificar las motivaciones o razones actuales por la solicitan o solicitarían un producto de nuestra cartera.

\subsubsection{Investigación cualitativa}

Cliente misterioso/ observación:

"La observación por pseudocompra o compra simulada es una técnica de investigación que permite evaluar y mejorar el nivel del servicio que ofrecen las empresas" (Nogales, 2004, p. 89). Este método no solo es utilizado para analizar la marca sino también a la competencia. En ese marco, se planea contratar a personas que simulen ser ejecutivos que quieren emprende una empresa mediana en la que se necesite software especializado similar al que ofrece Dicomtech. 


\section{Entrevista en profundidad}

Se utilizará la entrevista en profundidad, donde se planteará una guía de pautas a realizarse a personas expertas en software y que estén en la categoría. Esto con el motivo de rescatar las valoraciones que tienen los clientes respecto a la relación precio/calidad, motivaciones, creencias, actitudes, entre otros. Debido a que la dirección que toma la entrevista está determinada por las respuestas del sujeto y por el entrevistador, la libertad que confiere este método es ideal para los objetivos planteados

Se recomienda utilizar la técnica del escalonamiento, con ello se busca llegar a la red de significados del consumidor, de manera que se pueda indagar las profundas razones psicológicas y emocionales subyacentes de estos, las cuales afectan sus decisiones de compra (Malhotra, 2008). Otra razón que imperaría en Dicomtech para la utilización de este método es la dificultad para comprometer a una persona que tiene al mando personal, pues son ellos los decisores de compra. 


\section{CONCLUSIONES}

- La información correspondiente al mercado VARs en el Perú es nula o escasa impidiendo hacer un análisis más exhaustivo del mercado. De igual forma, La industria del software en el Perú no posee estudios significativos que ayuden con el propósito de conocer el mercado, incluso para inversores interesados

- El futuro de los VARs estará marcado por las tecnologías remotas, el cloud y las habilidades que se desarrollen entorno a estas. Como se mencionó, es probable que queden obsoletas en la cadena de distribución si no se ajustan con rapidez.

- Dicomtech hasta el momento está muy arraigado a las acciones de ManageEngine. Esto si bien consolida su posición le quita oportunidades de destacarse por sí solo. El manejo de la compañía apunta a una actitud reactiva más que a una proactiva.

- El universo de clientes que cumplen con los estándares de público objetivo de Dicomtech es bajo; a pesar de que año a año el número de empresas aumenta, el crecimiento será menor en los rubros de mediana/gran empresa y generará un estancamiento.

- Dentro de las razones que "impide" a Dicomtech subir su precio están los VARs puesto que muchos de ellos venden casi a precio fabricante y el hecho de que no se han comunicado debidamente los atributos diferenciadores de Dicomtech que en una comparación si justificaría un precio mayor.

- El principal canal de llegada a los clientes es el Email marketing, canal que ha servido para contactar y conseguir más clientes junto a las ya tradicionales llamadas telefónicas. Se ha tomado conocimiento que la construcción de la base de datos de clientes se dio por la asistencia a seminarios, ferias, realización de eventos y compra de bases de datos especializadas. 


\section{RECOMENDACIONES}

- Si bien el Core business es la comercialización de soluciones informáticas TI, se debe volver a hacer énfasis en los servicios como outsourcing para poder generar más ventas y ampliar el mercado.

- La elección de elementos para construir Brand Equity es adecuada, sin embargo, se recomienda hacer uso de más fondos para apoyar más el valor de marca usando los atributos aun sin explotar.

- Debido a que la competencia seguirá creciendo producto de los canales online, se recomienda un estudio más especializado de benchmarking e investigación del mercado que no impliquen gran inversión de recursos pero que den una mejor idea de la competencia y sus acciones

- Proponer a las instituciones del estado que realicen investigaciones sobre la industria TI que crece cada vez más en el Perú para poder contar con información que permite a las empresas desarrollarse de mejor manera. 


\section{REFERENCIAS}

ACG Research. (s.f.). Managed Service Cloud Opportunity. [Oportunidad de servicio gestionado en la nube]. [versión PDF] Recuperado de https://cdn.ttgtmedia.com/rms/pdf/module\%201.pdf

Alarcón, G. (9 de mayo de 2018). MTC cambiaría de nombre a Ministerio de Transportes y Tecnologías de la Información y la Comunicación. Gestión. Recuperado de https://gestion.pe/economia/mtc-cambiaria-nombre-ministerio-transportestecnologias-informacion-comunicacion-233218

AllBusiness Editors. (s.f.). What Is a Value-Added Reseller? [¿Qué es un revendedor de valor agregado?] Recuperado de https://www.allbusiness.com/what-is-a-value-addedreseller-1475-1.html

Apesoft: Con entidad dedicada a las TIC se duplicaría el crecimiento del sector software. (9 de Abril de 2017).Gestión. Recuperado de https://gestion.pe/tecnologia/apesoftentidad-dedicada-tic-duplicaria-crecimiento-sector-software-132697

Arciniega , A., Flores, C., y García, L. (2014). Integrando la oferta de las microempresas peruanas con la demanda de soluciones de software(tesis de maestría). [versión PDF]. Universidad Peruana de Ciencias Aplicadas. Recuperado de https://repositorioacademico.upc.edu.pe/bitstream/handle/10757/338547/Tesis\%20 Arciniega\%20-\%20Flores\%20-\%20Garc\%C3\%ADa.pdf?sequence=1yisAllowed=y

Arciniega , A., Flores, C., y García, L. (2014). Sectores de la Industria TI. [Tabla]. [versión PDF]. Recuperado de https://repositorioacademico.upc.edu.pe/bitstream/handle/10757/338547/Tesis\%20 Arciniega\%20-\%20Flores\%20-\%20Garc\%C3\%ADa.pdf?sequence=1yisAllowed=y

Base de datos TOP 100000. (2018). Ranking de empresas por facturacion. [Tabla].

Blackmon, K. (2017). Will VARs Have a Place in the 2018 Channel? [¿Tendrán los VAR un lugar en el canal de 2018?]. Channel Futures. Recuperado de https://www.channelfutures.com/channel-futures/will-vars-have-place-2018-channel

Custom Solutions Group. (s.f.). Time for Converged Infrastructure? [¿Tiempo para la infraestructura convergente?]. Recuperado de https://www.emc.com/collateral/white-papers/converged-infrastructure-wp.pdf

Decreto Supremo N 380-2017-EF. (23 de diciembre del 2017). El Peruano. Recuperado de http://www.gacetajuridica.com.pe/boletin-nvnet/ar-web/D.S380-2017-EF.pdf 
Dicomtech. (2018). Logo Dicomtech. [Figura]. Recuperado de https://www.dicomtech.com.pe/

Ferrel, O.yHartline, M. (2012). Estrategias de Marketing. Mexico: Cengage Learning.

Gillis, T. (5 de agosto de 2014). Where's the Value Going to Be in Value Added Resellers? [¿Dónde va a estar el valor en los revendedores de valor agregado?]. Forbes. Recuperado de https://www.forbes.com/sites/tomgillis/2014/08/05/wheres-thevalue-going-to-be-in-value-added-resellers/\#789b00ad4f36

Instituto Nacional de Estadística e Informática. (2015). Directorio Central de Empresas y Establecimiento. [versión PDF]. Recuperado de https://unstats.un.org/unsd/trade/events/2015/aguascalientes/9.-Panel\%20III\%20$\% 20$ Presentation\%201\%20-\%20INEI\%20Peru.pdf

Instituto Nacional de Estadística e Informática. (2015). Peru: Empresas, según segmento empresarial,2015-16. [Figura]. [versión PDF]. Recuperado de https://unstats.un.org/unsd/trade/events/2015/aguascalientes/9.-Panel\%20III\%20$\% 20$ Presentation\%201\%20-\%20INEI\%20Peru.pdf

Jones, D.y Roelevel, C. (2011). Key players in the software reseller market. [Jugadores clave en el mercado de revendedores de software]. [versión PDF]. Recuperado de https://www.insight.com/content/dam/insight/en_US/pdfs/insight/forrester/forresterInsight-cited-as-key-player-in-global-software-reseller-market-2011.pdf

Keller, K. L. (2008). Administracion Estrategica de marca, BRANDING. Naucalpan de Juárez, México: Pearson Educación.

Kotler, P.y Armstrong, G. (2007). MARKETING. Naucalpan de Juarez, México: Pearson Educacion.

Kotler, P.y Armstrong, G. (2007). Posibles propuestas de valor. [Tabla]. Naucalpan de Juarez, México: Pearson Educacion.

Kotler, P.y Keller, K. (2012). Dirección de marketing. Naucalpan de Juarez, Mexico: Pearson Educación.

Kotler, P.y Keller, K. (2016). Dirección de Marketing.México: Pearson.

MacLean, A. B. (2012). VALUE ADDED RESELLERS IN INFORMATION TECHNOLOGY: TRENDS. [VALOR AGREGADO DE REVENDEDORES EN TECNOLOGÍA DE LA INFORMACIÓN: TENDENCIAS.]. Recuperado de http://summit.sfu.ca/item/13028

Malhotra, N. K. (2008). Investigación de mercados. Naucalpan de Juárez, México: Person Educacion. 
ManageEngine. (2018). Precios de paquetes OpManager. [Figura]. Recuperado de https://store.manageengine.com/opmanager/

ManageEngine. (2018). Cartera de productos ManageEngine. [Tabla]. Recuperado de https://www.manageengine.com/productdocument.html

ManageEngine. (2018). Ejemplo de lanzamiento de producto. [Figura]. Recuperado de https://www.manageengine.com/productdocument.html

ManageEngine. (2018). Versiones y módulos de OpManager. [Figura]. Recuperado de https://www.manageengine.com/es/network-monitoring/opmanager-editions.html

ManageEngine. (s.f.). October Newsletter - 2018. [Boletín de octubre]. Recuperado de https://newsletter.manageengine.com/2018/10/17/october-newsletter-2018/

Nogales, Á. F. (2004). Investigacion y técnicas de mercado. Madrid: ESIC editorial.

Porter, M. (2008). Estrategia Competitiva: Técnicas para el análisis de los sectores industriales y de la competencia. México: Patria.

ProChile. (2013). Estudio de Mercado Servicio Industria del Software en Perú. [versión PDF]. Recuperado de https://www.prochile.gob.cl/wpcontent/files_mf/1380122114PMS_Peru_Software_2013.pdf

PROMPEX Perú y APESOFT. (2003). Situación de la industria nacional de software en el Perú. [versión PDF]. Recuperado de http://cendoc.esan.edu.pe/fulltext/edocuments/diagnosticosoftware2004_v3.pdf

Robbins, S.y Coulter, M. (2005). Administración. Naucalpan de Juarez, México: Pearson Educación.

Roy, G., Bova, T., Farre, T., Horowitz, A.y Harbert, T. (2008). Shift to managed services. [Cambio a servicios gestionados]. [versión PDF]. Connected, the link to the IT industry, 6-7. Recuperado de http://intl.imimages.com/upload/FTEN/files/Connected_Q108.pdf

Instituto Nacional de Estadística e Informática. (2016). PERÚ: Estructura Empresarial. [versión PDF]. Recuperado de https://www.inei.gob.pe/media/MenuRecursivo/publicaciones_digitales/Est/Lib144 5/libro.pdf

Santos Hernández, V. (2009). La industria del Software: Estudio a nivel global y América Latina. Recuperado de http://www.eumed.net/cursecon/ecolat/la/09/vsh.htm

Shapiro, B. P.y Bonoma, T. V. (mayo de 1984). How to segment Industrial Markets. [Cómo segmentar los mercados industriales]. Recuperado de https://hbr.org/1984/05/how-to-segment-industrial-markets. 
Shapiro, B. P.y Bonoma, T. V. (1984). Enfoque de Nido. [Figura]. Recuperado de https://hbr.org/1984/05/how-to-segment-industrial-markets

TechTarget (2018). Dos tipos de canales de distribucion .[Figura]. Recuperado de https://searchitchannel.techtarget.com/definition/VAR

Wheelen, T., y Hunger, J. D. (2007). Administracíón estratégica y política de negocios. Naucalpan de Juarez, México: Pearson Educación. 
ANEXOS 


\section{Anexo 1: Guía de indagación a expertos}

Buenos días / tardes mi nombre es . En esta oportunidad conversaremos sobre un tema interesante para usted como lo es soluciones para el área TI de su empresa.

Le recuerdo que no hay respuestas buenas o malas, correctas o incorrectas. Todas sus respuestas son importantes.

\section{ASPECTOS GENERALES}

*Para fines del estudio se tomarán en cuenta solo los productos relacionados a Active Directory, Help Desk y Seguridad de TI que son los de mayores ventas.

Objetivos:

- Explorar los softwares que ha adquirido/usado, sus funcionalidades, proveedor, características, razón de compra, etc.

- Indagar en la imagen y posicionamiento de los proveedores actuales

- Profundizar en la fortalezas y debilidades de los proveedores y competidores actuales

- Sondear en las oportunidades de mejora y sugerencias para que los proveedores actuales brindes un mejor servicio

- Indagar en los problemas que ha tenido en el pasado con los proveedores

- Explorar los atributos que buscan en un proveedor de software (antigüedad de la empresa, cartera de software,

01. ¿Qué software ha adquirido o usado últimamente, nos puede comentar sobre algunas características del software?

02. ¿Porque lo adquirió? ¿piensa que el software tenía alguna característica en particular que sobresaliera de otras ofertas?

03. ¿En qué medida cree usted que el proveedor del software influye en su decisión final?

04. ¿Conoce los VARs? ( explicarlo)

05. ¿Qué opinión tiene al respecto de los VARs en el Perú?

06. ¿Hay alguno que para usted destaque?

07. ¿Cuán importante cree que son los VARs en la cadena de distribución? 


\section{Anexo 1: Guía de indagación a expertos}

08. Sabiendo lo que es un VAR, ¿cuáles cree que son las ventajas y desventajas de serlo?

09. ¿Qué opinión tiene de los servicios que ofrecen los VARs?

10. ¿Qué cree usted que deberían mejoran los proveedores actuales?

11. ¿Conoce alguna tendencia que deberían adoptar?

12. ¿Cree usted que se está desperdiciando alguna oportunidad de mercado?

13. ¿Cómo cree que será el futuro para los VARs?

14. ¿Ha tenido usted algún inconveniente con algún software que le vendieron? ¿El inconveniente ha sido con el proveedor o con el fabricante?

15. ¿Ha tenido algún problema particular con algún proveedor? (VARs), profundizar

16. ¿Que busco o busca a la hora de adquirir un software

17. ¿Cuáles cree que son los atributos más importantes para seleccionar al proveedor? (Antigüedad, empresa, cartera)

18. ¿Cuánto afecta el precio en su decisión final?

19. ¿Cuánto afecta la calidad en su decisión final?

20. ¿Cuánto afecta la cadena de mando en su decisión final? 


\section{Anexo 2: Guión y pautas de Mystery Shopper.}

El Mystery Shopper realiza ciertas actividades durante la interacción con las piezas de marketing o el proceso de compra. Para esto se ha establecido una serie de pasos sin orden que puede realizar el mystery shopper para cumplir con los objetivos planteados.

Objetivos

- Determinar las principales diferencias entre la oferta de la competencia y la de Dicomtech

- Determinar cómo se construye la relación cliente- marca

- Averiguar los testimoniales de mayor relevancia

- Identificar la propuesta de valor de la competencia

- Evaluar las actividades digitales y presenciales que realiza la competencia para atraer clientes

- Valorar el servicio que ofrece la competencia

- Comprender su posición actual

Actividades por internet:

- Registrarse en formularios de producto

- Efectuar consultas en webinars

- Expresar alguna queja

- Adquirir o pretender un producto

- Registrarse en una capacitación

- Visibilizar la disconformidad hacia la marca en algún sitio web

- Provocar alguna reacción negativa

Actividades por teléfono:

- Hacerse pasar por una empresa nueva

- Pactar una demostración de producto

- Preguntar cotizaciones 


\section{Anexo 2: Guión y pautas de Mystery Shopper.}

- Efectuar quejas falsas

- Efectuar preguntas sobre clientes que posean

- Preguntar sobre procesos de instalación, mantenimiento, renovación, entre otros

- Plantear problemas falsos

Actividades Presenciales

Evaluar activaciones de marca y relaciones públicas de la competencia, en este caso se busca que el Mystery Shopper interactúe con algún representante de la competencia a fin de poner a prueba sus procesos. 


\section{Anexo 3: Cotización SolarWinds}

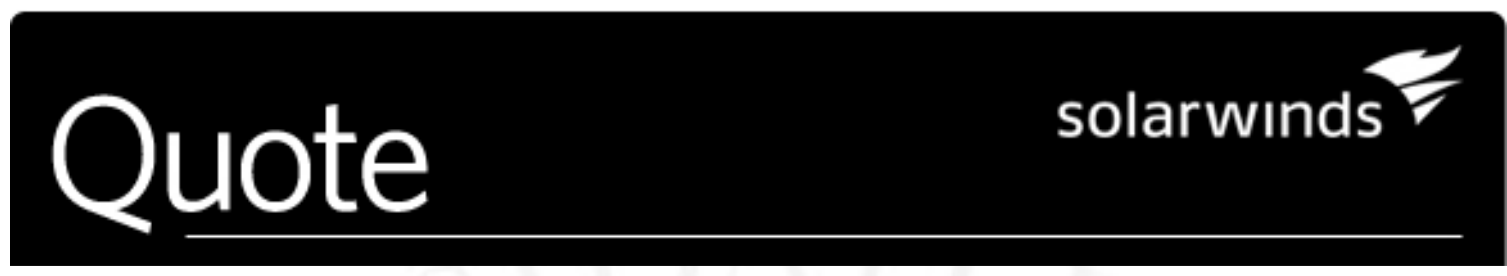

\section{This quote is valid until 02/12/2018}

Pleasedirectallquestions, includingterms ofsales andpromotions, viaemailcorrespondenceto sales@solarwinds.com.

\begin{tabular}{|l|l|}
\hline SolarWinds Quote \#209293 & November 02, 2018 \\
\hline Name: ivo carlos & SolarWinds Software Europe Limited \\
Company: & EMEA Headquarters \\
DICOMTECH & PO BOX 730720 \\
Dallas United \\
Location: Peru, & States Phone: \\
Email: ivocarlosramos@gmail.com & 866.530 .8100 \\
Phone: 978271586 & Fax: 512.682.9301 \\
& VAT ID: IE9652586C \\
& $\underline{\text { http://www.solarwinds.com }}$ \\
& $\underline{\text { sales@ solarwinds.com }}$ \\
\hline
\end{tabular}

Qty ProductID / Description

1247 - SolarWinds Network Performance Monitor SL100 (up to 100 elements) - License with 1st-year Maintenance
Unit List

Price

Sub-Total

$$
\$ 2,955
$$

$\$ 2,955$

Quotes are validfor 30 days, with the exception of sale pricing. Sale pricing expires as noted when the promotionends. Thisquote isfor informative purposes, is not a formal offer of goods or services, andis not a bindingcontract. 